\title{
Influence of TIMP3/SYN3 polymorphisms on the phenotypic presentation of age-related macular degeneration
}

\author{
Daniel Ardeljan ${ }^{1}$, Catherine B Meyerle², Elvira Agron², Jie Jin Wang ${ }^{3}$, Paul Mitchell ${ }^{3}$, Emily Y Chew ${ }^{2}$, \\ Jing Zhao ${ }^{4}$, Arvydas Maminishkis ${ }^{4}$, Chi-Chao Chan ${ }^{1}$ and Jingsheng Tuo ${ }^{\star, 1}$
}

Age-related macular degeneration (AMD) is a leading cause of irreversible central visual loss in the elderly. A recent genomewide association studies (GWAS) reported that rs9621532 near the tissue inhibitor of metalloproteinase 3 (TIMP3)/synapsin III (SYN3) region of 22q12.3 is associated with AMD. In this study, we characterize its phenotypic influence on AMD using three independent study cohorts: case-control studies from the National Eye Institute Clinical Center (NEI, $n=397$ ) and the AgeRelated Eye Disease Study $(n=523)$ as well as a nested case-control study from Blue Mountains Eye Study (BMES, $n=852$ ). Comparisons between cases and controls show no association between rs9621532 and AMD in the three sample sets. However, stratifying NEI cases uncovers a moderate protective role of rs9621532 in neovascular AMD (nAMD) and the association adhered to a dominant model (odds ratios $=0.32 ; 95 \% \mathrm{Cl}: 0.11-0.89 ; P=0.02$ ). The BMES data followed the same pattern of association with nAMD as that seen in the NEI sample but did not reach statistical significance. Polychotomous logistic regression showed a trend that rs9621532 correlates with less severe disease, for example, with the majority of carriers having intermediate AMD rather than nAMD/geographic atrophy AMD. Functionally, rs9621532 influences TIMP3 mRNA expression in cultured primary human fetal retinal pigment epithelium (hfRPE) cells. In hfRPE donors carrying the protective rs9625132 allele, we measured a reduction in TIMP3 mRNA by quantitative RT-PCR. Our data suggest that rs 9621532 carriers have a lower risk of developing nAMD, potentially because of decreased transcription of TIMP3.

European Journal of Human Genetics (2013) 21, 1152-1157; doi:10.1038/ejhg.2013.14; published online 20 February 2013

Keywords: age-related macular degeneration; TIMP3; phenotype; single-nucleotide polymorphism; Blue Mountains Eye Study; Age-Related Eye Diseases Study

\section{INTRODUCTION}

Age-related macular degeneration (AMD) is a prevalent cause of central blindness in people of European ancestry over the age of $50{ }^{1}$ Epidemiological studies show that the development of AMD is a multifactorial process including both genetic and environmental risk factors. Environmental factors such as age, body mass index, and exposure to cigarette smoking contribute to the risk of developing AMD. $^{2-6}$ In addition to these factors, various single-nucleotide polymorphisms (SNPs) have been widely reported to be associated with AMD. ${ }^{7}$ Genome-wide association studies (GWAS) have confirmed multiple AMD-associated loci on chromosomes 1 and 10, including the genes $C F H, C 2, C 3, C F B, A R M S 2$, and HTRA1. Recently, a large-scale GWAS with thousands of cases and controls reported several additional AMD-associated loci, including rs9621532 near the tissue inhibitor of metalloproteinase 3 (TIMP3) and synapsin III (SYN3) region of chromosome 22q12.3..$^{8,9}$ The TIMP3 gene is located within an intron of SYN3 and is transcribed in the opposite direction. The SNP is located approximately 100-kb upstream of TIMP3 within the same intron of SYN3 (Figure 1a).

As AMD progresses to its advanced stages, it can be classified as either neovascular AMD (nAMD) or geographic atrophy (GA).
Central vision loss is gradual in GA because of slow atrophy of photoreceptors and retinal pigment epithelium (RPE), whereas nAMD patients can lose central vision rapidly because of the development of choroidal neovascularization (CNV) and fluid leakage or hemorrhage. These marked differences in disease phenotype may be caused by genetic variations, which serve as bifurcation points from which a patient with intermediate stage disease may be more or less likely to develop nAMD and/or GA. For example, studies have suggested that SNPs of the CFH and ARMS2/HTRA1 differentially affect phenotypic outcome within AMD cohorts, particularly for lesion size, change in RPE pigmentation, risk for large drusen, among others. ${ }^{10,11}$ TIMP3 is a senescence protein that regulates extracellular matrix remodeling and is thought to maintain the integrity of Bruch's membrane. ${ }^{12,13}$ TIMP3 has been shown to suppress angiogenesis via competitive inhibition of binding between vascular endothelial growth factor (VEGF) and VEGF receptor-2 (VEGFR-2). ${ }^{14}$ Considering these data, we speculate that SNPs near the TIMP3/ SYN3 locus could be associated with risk of nAMD.

In this study, we used three well-established independent AMD sample sets to analyze genotype frequencies in TIMP3/SYN3 rs9621532 and additional SNPs within the region (Table 1).

${ }^{1}$ Laboratory of Immunology, National Eye Institute, National Institutes of Health, Bethesda, MD, USA; ${ }^{2}$ Division of Epidemiology and Clinical Applications, National Eye Institute, National Institutes of Health, Bethesda, MD, USA; ${ }^{3}$ Centre for Vision Research, Department of Ophthalmology and Westmead Millennium Institute, University of Sydney, Westmead, Australia; ${ }^{4}$ Section of Epithelial and Retinal Physiology and Disease, National Eye Institute, National Institutes of Health, Bethesda, MD, USA

${ }^{*}$ Correspondence: Dr J Tuo, Laboratory of Immunology, National Eye Institute, National Institutes of Health, 10/10N103, 10 Center Drive, Bethesda, MD, 20892-1857, USA Tel: + 1301496 0417; Fax: + 1301402 8664; E-mail: tuoj@nei.nih.gov

Received 16 July 2012; revised 10 December 2012; accepted 16 January 2013; published online 20 February 2013 

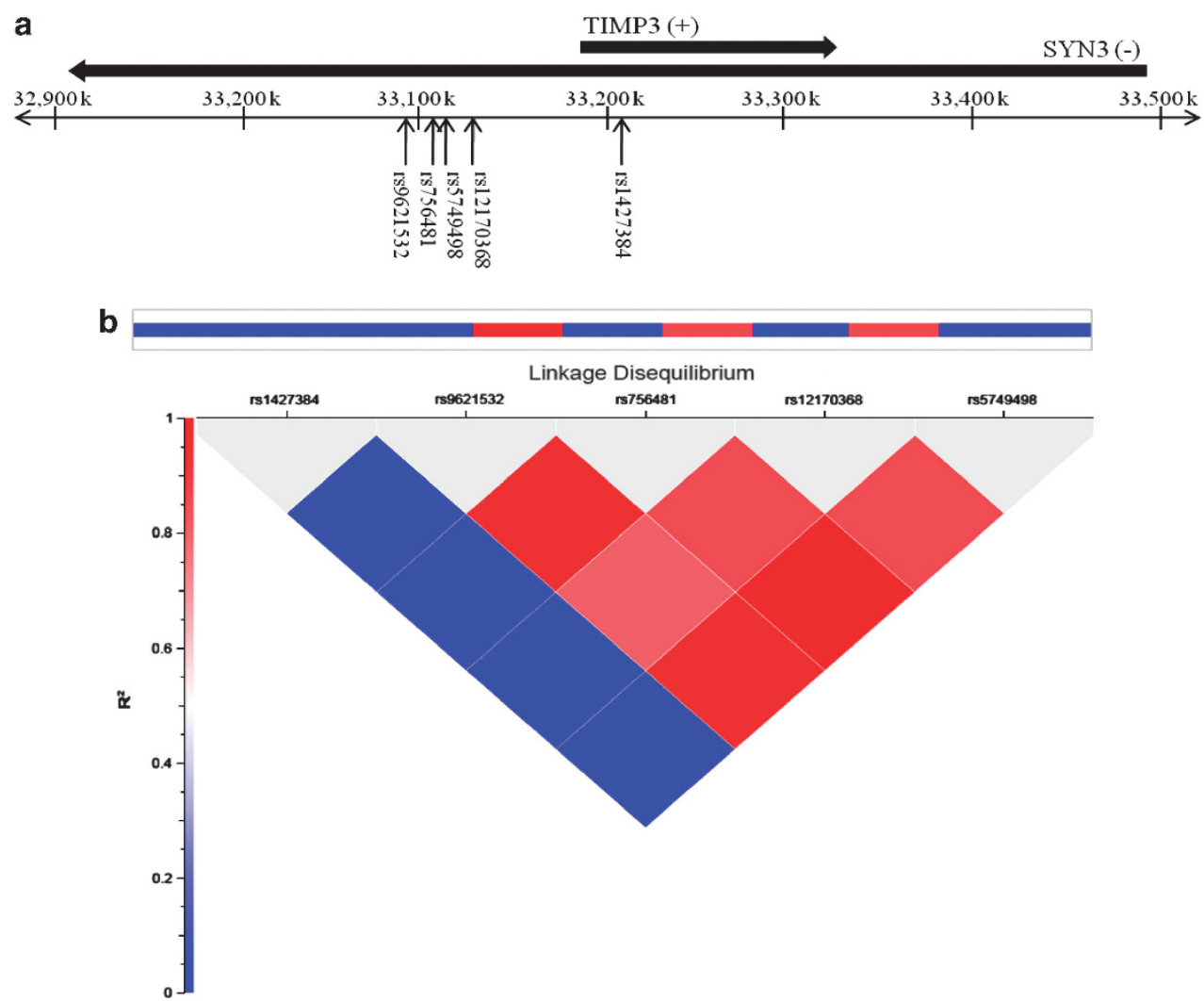

Figure 1 The TIMP3/SYN3 genomic neighborhood. (a) Shows the relative positions of the five tested SNPs with respect to each other and the TIMP3 and SYN3 genes on 22q12.3. (b) Shows the degree of LD among the five tested SNPs.

Table 1 Demographic information of participants in three sample sets used in this study

\begin{tabular}{|c|c|c|c|c|c|c|c|c|}
\hline Sample set & Cohort & $N$ & Age $(m e a n \pm S D)$ & Ever smokers no. (\%) & Female no. (\%) & Advanced AMD no. (\%) & GA no. (\%) & nAMD no. (\%) \\
\hline \multirow[t]{2}{*}{$\mathrm{NEI}$} & Control & 160 & $73.9 \pm 10.8$ & $24(15.0)$ & $92(57.5)$ & 0 & 0 & 0 \\
\hline & AMD & 237 & $79.0 \pm 8.1$ & $122(51.5)$ & $126(53.2)$ & $204(86.1)$ & $45(19.0)$ & $159(67.1)$ \\
\hline \multirow[t]{2}{*}{ AREDS } & Control & 193 & $76.8 \pm 4.3$ & $99(51.3)$ & $105(54.4)$ & 0 & 0 & 0 \\
\hline & AMD & 330 & $79.2 \pm 5.1$ & $190(57.6)$ & $195(59.1)$ & $279(84.5)$ & $124(37.5)$ & $155(47.0)$ \\
\hline \multirow[t]{2}{*}{ BMES } & Control & 568 & $75.1 \pm 7.4$ & $54(9.5)$ & $344(60.6)$ & 0 & 0 & 0 \\
\hline & AMD & 284 & $75.3 \pm 7.6$ & $27(9.5)$ & $172(60.6)$ & $54(19.0)$ & $20(7.0)$ & $34(22.2)$ \\
\hline
\end{tabular}

Abbreviations: AREDS, Age-Related Eye Disease Study; BMES, Blue Mountains Eye Study; NEI, National Eye Institute Clinical Center.

Association of the TIMP3/SYN3 region with AMD was assessed by phenotypic stratification, haplotype block association, and polychotomous regression with respect to disease severity. We conducted a functional assay to uncover any correlation between rs9621532 genotype and TIMP3/SYN3 mRNA expression using cultured primary human fetal RPE (hfRPE) from donors with different rs9621532 genotypes.

\section{MATERIALS AND METHODS}

\section{Study subjects}

This research followed the tenets of the Declaration of Helsinki and the study protocol was approved by IRBs. All participants gave informed consent. The three study group populations are summarized in Table 1. Methods for participant selection and clinical evaluation of subjects in the National Eye Institute Clinical Center (NEI) and the Age-Related Eye Disease Study (AREDS) studies have been previously defined and briefly described below. ${ }^{2,4,15}$
AMD patients and control subjects from NEI and AREDS studies were evaluated by NEI ophthalmologists using the AREDS criteria, although none of the subjects within these two cohorts overlapped. ${ }^{3,15}$ AMD cases were confirmed by independent grading of fundus photographs. All controls presented either no drusen or $<5$ small drusen $(<63 \mu \mathrm{m}$ in diameter) and no signs of other retinal diseases including but not limited to high myopia, retinal dystrophies, central serous retinopathy, vein occlusion, diabetic retinopathy, or uveitis. DNA samples for this study were selected from NEI and AREDS participants who self-identified as non-hispanic white.

The Blue Mountains Eye Study (BMES) is a population-based cohort study of common eye diseases and health-related parameters among suburban residents aged 49 years or older in the Blue Mountains region west of Sydney, Australia. ${ }^{16-18}$ Nested samples from the BMES population included 852 subjects, which can be subdivided into 'case' and 'control' groups. In this study, the 'case' group was composed of a combination of patients with early AMD (230 subjects) and late AMD (20 GA and 34 nAMD subjects). Early AMD was defined as the presence in either eye of: (1) large ( $125 \mu \mathrm{m}$ or larger in diameter), soft, indistinct, or reticular drusen within the macular area or (2) presence of large, soft, distinct drusen within the macula in addition to retinal 
pigmentary abnormalities. ${ }^{17}$ GA was diagnosed when a patient's retina was affected in a discrete area greater than at least $175 \mu \mathrm{m}$ in diameter and which was characterized by a sharp border in addition to the presence of visible choroidal vessels. nAMD was defined as serous or hemorrhagic detachment of the sensory retina or RPE, the presence of subretinal or sub-RPE hemorrhages, or subretinal fibrous scarring. The BMES 'control' group was matched with the case group on age, sex, and smoking status. Control participants were defined as having no signs of early AMD in the macular area. ${ }^{17}$

\section{SNP selection and genotyping}

We selected rs9621532 because it was reported to be associated with AMD. ${ }^{8,9}$ Three additional tag-SNPs were selected in a $100-\mathrm{kb}$ region spanning rs9621532. To find the three SNPs, we set the minor allele frequency cutoff to 0.01 and $R^{2}$ linkage disequilibrium (LD) with rs9621532 >0.8. rs1427384 in the $3^{\prime}$-UTR region of TIMP3 was also included in this study based on silicon analysis, which indicates the SNP is located in a microRNA-binding site (SNP function prediction, http://snpinfo.niehs.nih.gov/snpfunc.htm). Figure 1 illustrates the location of the SNPs on chromosome 22q12.3 and details the LD block.

SNP genotyping was performed using the Taqman SNP Genotyping Assay from ABI (Applied Biosystems, Foster City, CA, USA). All the assays were inventory stocks save for rs9621532, which was custom designed.

\section{Transcript/mRNA expression of TIMP3 and SYN3 in hfRPE}

Fetal eyes were obtained from Advanced Bioscience Resources (Alameda, CA, USA) at 16-18 weeks of gestation. The separation and culture of primary hfRPE were reported previously. ${ }^{19}$ This study included hfRPE from 32 donors.

Genomic DNA was extracted from hfRPE to identify the genotypes at rs9621532 from each donor. Total RNA from hfRPE was extracted using Trizol (Invitrogen, Carlsbad, CA, USA). cDNA was synthesized by reverse transcriptase (Taqman reverse transcription reagents, Applied Biosystems). The primers/probes for TIMP3 and SYN3 were purchased from Applied Biosystems as inventoried TaqMan gene expression reagents. Relative quantitative realtime PCR was performed to determine the CT values by the $2^{-\Delta \Delta \mathrm{Ct}}$ analysis method using GAPDH as an endogenous control. The relative expression of TIMP3 and SYN3 in rs9621532 minor-allele carriers (heterozygotes) was compared with that from rs9621532 homozygous major-allele carriers. Each sample was analyzed in duplicate in three independent experiments.

\section{Statistical analysis}

The power to detect effects of SNP variants on intermediate and advanced AMD was calculated using the binomial distribution. SNP allelic association and genotypic association as a dominant model (carriers of at least one minor allele versus those with two major alleles) was analyzed using logistic regression in which the case-versus-control status was designated as the outcome and was adjusted for age, gender, and smoking status. $P$-values, odds ratios (OR) of association, and Hardy-Weinberg Equilibrium (HWE) $P$-value were computed using a $\chi^{2}$ test. Multiple testing was corrected by the false discovery rate (FDR) and by setting permutation to analyze 1000 simulations. ${ }^{20}$ Haplotypes were decided based on the LD scores of tested SNPs and haplotype association was estimated by a $\chi^{2}$ test. Associations with AMD severity and genotypes were evaluated using polychotomous logistic regression, which considered intermediate stage AMD and advanced AMD as distinct categories.
Association analyses were performed using SVS software (version 7.4.1; HelixTree Genetics Analysis Software, Golden Helix Inc., Bozeman, MT, USA; htpp://www.goldenhelix.com/SNP_Variation/HelixTree/index.html). The means for mRNA expression of TIMP3 and SYN3 were compared using Student's $t$-test. Polychotomous logistic regression was computed by SAS software (ver. 9.1; SAS Institute, Cary, NC, USA). Differences were considered significant when $P<0.05$ in testing.

\section{RESULTS}

\section{Statistical power analysis}

We used published rs9621532-AMD association data as a reference for power analysis. ${ }^{8}$ Based on a pre-defined two-sided alpha of 0.05 , there was $>95 \%$ power to detect a $\pm 3 \%$ departure from an rs 9621532 allele frequency of $5.7 \%$ in each of the three sample sets. Study power remained $>80 \%$ after stratifying the cases to nAMD in the NEI and AREDS cohorts but not in BMES.

\section{rs9621532 is associated with nAMD}

The results of SNP associations by logistic regression analysis of AMD patients and controls in the NEI sample set are shown in Table 2. None of the five tested SNPs were associated with AMD as a whole in the NEI (Table 2) or in the AREDS and BMES sets (rs9621532 data in Table 4). Exclusion of all non-nAMD cases from the NEI cohort revealed a significant association between SNPs rs9621532, rs756481, and rs5749498 with nAMD $(P<0.05)$. Importantly, the allelic frequencies at all five SNPs were within the boundaries of HWE $(P>0.05)$ in the NEI sample set.

We focused on the influence of rs9621532, which exhibited the strongest association with nAMD among the five SNPs tested, and found that the minor $\mathrm{C}$ allele associated with the nAMD phenotype following a dominant model (Table 3, $P=0.023$; OR $=0.315 ; 95 \%$ CI: 0.112-0.891) and suggested a protective role from development of nAMD. This association remained significant $(P=0.034 ;, \mathrm{OR}=0.32)$ after adjusting for confounding factors including smoking, age, and gender, or after correcting for multiple testing by calculating the FDR $(P=0.034)$. We confirmed that rs9621532, rs746481, rs5749498, and rs12170368 are in LD, evidenced by a high $R^{2}$ value (Figure $1 \mathrm{~b}$ ), and then evaluated the minor allele haplotype block for all four SNPs (CGTT), observing an even stronger protective association with nAMD (Table 3, $P=0.011$; OR $=0.259$; 95\% CI: 0.085-0.789).

Dominant model analysis on AREDS and BMES data sets separately failed to detect any significant associations between rs9621532 with AMD as a whole or specifically with nAMD (Table 4). Interestingly, a nAMD OR of 1.256 for rs9621532 in AREDS cases suggested a harmful effect of the minor $\mathrm{C}$ allele, but we found that rs9621532 frequencies deviated from HWE in the AREDS control group (Table $4, P=9.82 \times 10^{-5}$ ), thus rendering the set biased for use in the analysis of this particular SNP. In the BMES sample set, we were able to deduce a protective trend for the minor $\mathrm{C}$

Table 2 Distribution of minor allele of tested TIMP3 SNPs and their association with AMD in the NEI sample set

\begin{tabular}{|c|c|c|c|c|c|c|c|c|c|}
\hline SNP minor/major & $\begin{array}{c}\text { Control } \\
\text { minor/major } \\
(\%)\end{array}$ & $\begin{array}{c}\text { All cases } \\
\text { minor/major } \\
\text { (\%) }\end{array}$ & $P$-value & OR $(95 \% \mathrm{Cl})$ & $\begin{array}{c}n A M D \\
\text { minor/major } \\
\text { (\%) }\end{array}$ & $P$-value & OR $(95 \% \mathrm{Cl})$ & FDR & $\begin{array}{c}\text { HWE P of } \\
\text { control }\end{array}$ \\
\hline rs9621532 A/C & 15/305 (4.69) & $15 / 453(3.21)$ & 0.286 & $0.67(0.32-1.40)$ & $5 / 321(1.53)$ & 0.025 & $0.33(0.12-0.91)$ & 0.075 & 0.534 \\
\hline rs756481 A/G & 15/301 (4.75) & $14 / 458(2.97)$ & 0.193 & $0.61(0.29-1.29)$ & 6/310 (1.90) & 0.046 & $0.39(0.15-1.01)$ & 0.092 & 0.531 \\
\hline rs5749498 G/T & 15/301 (4.75) & $14 / 454$ (2.99) & 0.201 & $0.61(0.29-1.30)$ & $6 / 310(1.90)$ & 0.046 & $0.39(0.15-1.01)$ & 0.092 & 0.531 \\
\hline rs12170368 C/T & $15 / 295(4.84)$ & $14 / 450(3.02)$ & 0.191 & $0.61(0.29-1.29)$ & 7/305 (2.24) & 0.080 & $0.45(0.18-1.12)$ & 0.120 & 0.527 \\
\hline rs1427384 A/G & $67 / 219(23.43)$ & $128 / 314(23.43)$ & 0.100 & $1.33(0.95-1.88)$ & $89 / 211$ (29.67) & 0.088 & 1.37 (0.95-1.99) & 0.117 & 0.389 \\
\hline
\end{tabular}


Table 3 Dominant model analysis of nAMD in the NEI sample set

\begin{tabular}{lccccc}
\hline & Genotype & Control, $N(\%)$ & $n A M D, N(\%)$ & $P / P_{a}$ & OR (95\% CI) \\
\hline rs9621532 & AA & $145(90.6)$ & $153(96.8)$ & $0.023 / 0.034$ & $0.315(0.112-0.891)$ \\
Haplotype block & AC + CC & $15(9.38)$ & $5(3.2)$ & & $0.034 /$ ND \\
\hline
\end{tabular}

Abbreviations: FDR, false discovery rate to adjust for multiple testing; ND, no data.

Both rs9621532 and its haplotype block (rs9621532, rs756481, rs5749498, and rs12170368) are considered for association with NV AMD where possessing one or two of the minor alleles confers protection from disease. $P$-values are adjusted for age, gender, and smoking status $\left(P_{\mathrm{a}}\right)$.

Table 4 Dominant model analysis of rs9621532 in all AMD and nAMD in the AREDS and BMES sample sets

\begin{tabular}{|c|c|c|c|c|c|c|c|c|c|}
\hline \multirow[b]{2}{*}{ Sample } & \multirow[b]{2}{*}{ Genotype } & \multicolumn{2}{|c|}{ Control } & \multicolumn{3}{|c|}{$A M D$} & \multicolumn{3}{|c|}{$n A M D$} \\
\hline & & $N \%$ & HWE P & $N \%$ & $P$-value & OR $(95 \% \mathrm{Cl})$ & $N \%$ & $P$-value & OR $(95 \% \mathrm{Cl})$ \\
\hline \multirow[t]{2}{*}{ AREDS } & AA & $177(91.7)$ & $9.82 \times 10^{-5}$ & $299(90.6)$ & 0.670 & $1.147(0.610-2.156)$ & $185(89.8)$ & 0.512 & $1.256(0.635-2.484)$ \\
\hline & $A C+C C$ & $16(8.3)$ & & $31(9.4)$ & & & $21(10.2)$ & & \\
\hline \multirow[t]{2}{*}{ BMES } & AA & $503(89.7)$ & 0.640 & $254(92.0)$ & 0.273 & $0.751(0.450-1.255)$ & $32(94.1)$ & 0.402 & $0.452(0.127-2.320)$ \\
\hline & $A C+C C$ & $58(10.3)$ & & $22(8.0)$ & & & $2(5.9)$ & & \\
\hline
\end{tabular}

Table 5 Polychotomous logistic regression of AMD grade with rs9621532 in a dominant model

\begin{tabular}{|c|c|c|c|c|c|c|c|}
\hline \multirow[b]{3}{*}{ Sample set } & \multirow[b]{3}{*}{ Genotype } & \multicolumn{6}{|c|}{ Disease grade } \\
\hline & & \multicolumn{3}{|c|}{ Intermediate } & \multicolumn{3}{|c|}{$n A M D+G A$} \\
\hline & & $\%$ & OR $(95 \% \mathrm{Cl})$ & $P$-value & $\%$ & OR $(95 \% \mathrm{Cl})$ & $P$-value \\
\hline NEI & $A C+C C$ & 8.3 & $0.86(0.24-3.15)$ & 0.821 & 4.6 & $0.46(0.19-1.07)$ & 0.072 \\
\hline BMES & $A C+C C$ & 8.6 & $0.81(0.47-1.40)$ & 0.452 & 5.6 & $0.51(0.15-1.69)$ & 0.270 \\
\hline
\end{tabular}

allele at rs9621532 from nAMD with an OR of 0.45 (Table 4). However, a lack of power for statistical testing because of the small number of late AMD cases in the BMES meant that the association could not reach significance.

The C allele of rs9621532 trends toward less severe disease Table 5 details the results of polychotomous logistic regression on disease grade in NEI and BMES sample sets separately. For each set, the analysis was performed following a dominant model wherein possession of at least one $\mathrm{C}$ allele was deemed to confer protection. We observed a trend in the NEI and BMES showing lower frequencies of $\mathrm{C}$ allele carriers in the advanced AMD category than in the intermediate AMD group (Table 4).

rs9621532 C carriers express lower TIMP3 and SYN3 in RPE As rs9621532 is associated with disease and yet located in non-coding regions of both TIMP3 and SYN3, we evaluated the effect of the allelic variation on the expression of the two genes in ocular tissue. We genotyped hfRPE cells from 32 donors and found 4 to be heterozygous (AC) and 28 to be homozygous for the major allele (AA) at rs9621532. We selected six homozygotes at random and the four heterozygotes to compare the relative mRNA expression of TIMP3 and SYN3 by the Student's $t$-test. There was approximately $65 \%$ lower TIMP3 $(P<0.05)$ and $34 \%$ lower SYN3 $(P>0.05)$ expression in heterozygotes with respect to homozygotes (Figure 2 ).

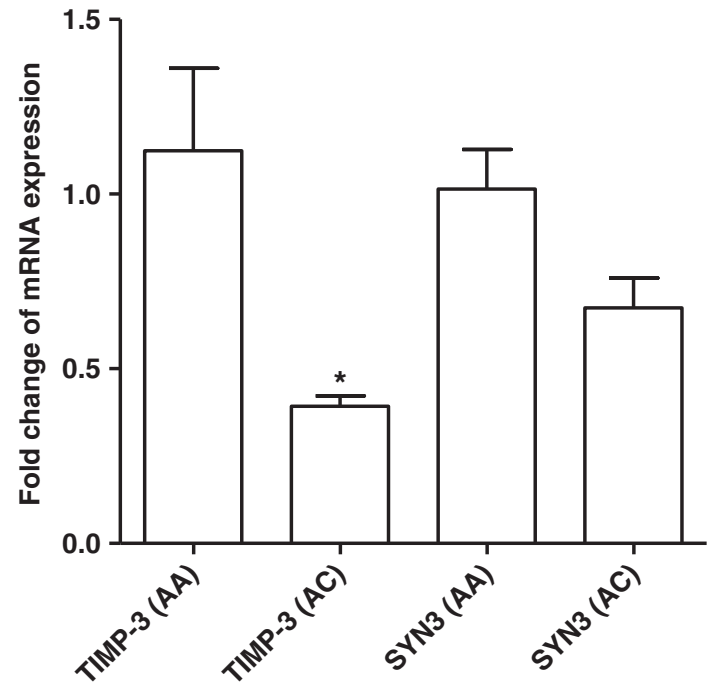

Figure 2 TIMP3 and SYN3 mRNA expression in hfRPE as a function of rs9621532 genotype. Error bars indicate SD. ${ }^{*} P<0.05$.

\section{DISCUSSION}

We had three goals in this study: (1) to replicate the findings that the SNP rs9625132 was associated with development of AMD and to extend this analysis to SNPs that are in LD with rs9621532, (2) to 
analyze the influence that rs9621532 and the additional SNPs contribute to AMD phenotypes, and (3) to correlate this genetic variation to TIMP3/SYN3 gene expression.

In three well-established, independent sample sets, referred to as the NEI, AREDS, and BMES cohorts, the association between SNP rs9621532 and AMD at-large did not reach statistical significance in any of the tested SNPs. However, stratifying the cases by disease phenotype revealed a statistically significant difference in rs9621532 allele frequencies within the NEI nAMD cases after adjusting for age, gender, and smoking status. The association demonstrated a protective role and followed a dominant model. Although the haplotype block consisting of the four minor alleles from rs9621532, rs756481, rs5749498, and rs12170368 provided the greatest and most significant protection from nAMD, rs9621532 was the most significant individual SNP.

In the BMES cohort, the association with nAMD failed to reach significance because of limitations resulting from a very small sample size of the advanced cases. This is due to the design of the BMES as a population-based prospective study, which features only 34 nAMD cases. In the AREDS cohort, the minor rs9621532 allele suggested increased susceptibility to nAMD. On closer analysis, we found that the rs9621532 genotypic distribution within the AREDS control group departed significantly from HWE and might result from possible population stratification. One expects a disease population to diverge from HWE because only those individuals with the proper genetic influences are 'selected' for the disease phenotype. However, control participants are not suffering from AMD and thus their allelic frequencies must be in HWE in order to detect influential association. This renders the AREDS sample set unsuitable for analysis of rs9621532.

To date, it has not been proven whether the variants at rs9621532 influence the development of AMD by altering TIMP3 or SYN3 in quality or quantity. We tested whether genotype contributes to a change in gene expression and found that in hfRPE, C carriers at rs9621532 expressed 65\% less TIMP3 and 34\% less SYN3. Our results indicated that rs9621532 significantly reduced transcriptional output at the TIMP3 locus. We were unable to measure the effects of the homozygous minor allele genotype, CC, because of the allele's low frequency in the population and the highly limited availability of hfRPE donor lines.

Physiologically, TIMPs inhibit matrix metalloproteinases, a group of peptidases involved in the degradation of extracellular matrix (ECM). TIMP3, primarily found within ECM of epithelial cells in eyes, lungs, and kidneys, ${ }^{12,21}$ is highly expressed in RPE. ${ }^{22}$ Mutations in TIMP3 were implicated in Sorsby's fundus dystrophy (SFD), an autosomal dominant disorder featuring accumulation of macular drusen and progression to CNV and retinal degeneration. ${ }^{23-26}$ In SFD as well as in the TIMP3-independent dystrophic disease retinitis pigmentosa, accumulation of TIMP3 was observed by immunohistochemistry, highlighting the importance of ECM turnover in the pathologic state. ${ }^{27}$ At the same time, SFD is known to be caused by missense mutations within TIMP3 exons, which are likely to alter ECM integrity qualitatively by either changing TIMP3 binding specificity or affinity for its substrates. ${ }^{28}$ Alternatively, changes in quantity of TIMP3 expression and activation have been shown to alter intracellular signaling of the MAPK pathways, suggesting that imbalances in ECM remodeling induce important cell regulatory pathways. ${ }^{29}$ Aberrant MAPK signaling has been recently implicated in AMD pathology. ${ }^{30}$ Increased TIMP3 protein in Bruch's membrane and drusen of AMD eyes is also correlated with AMD disease pathology. ${ }^{13}$ Besides regulating ECM remodeling, ${ }^{31}$
TIMP3 serves as an extracellular ligand for VEGFR-2 and thus directly regulates angiogenesis. ${ }^{14}$ Most importantly, TIMP3 protein is undetectable in RPE in young individuals but increases in intensity in older individuals ${ }^{12}$ and significantly elevated TIMPs were also detected in Bruch's membrane of AMD patients with respect to agematched controls. ${ }^{13}$ Enhanced expression of TIMP3 could prevent ECM remodeling and contribute to a thickening Bruch's membrane with lower integrity because of changes in the homeostatic balance of turnover, which is precisely what is observed in AMD. ${ }^{13}$ In contrast to SFD in which disease has early onset because of qualitative changes in TIMP3, in AMD the contribution to disease stems more likely from long-term sequestration of TIMP3 within Bruch's membrane. The relationship between TIMP3 and SYN3 is still unclear.

In conclusion, our data suggest that rs9621532 carriers have a lower risk of developing $\mathrm{nAMD}$, potentially because of decreased transcription of TIMP3. We stress that the association between rs9621532 and nAMD is a moderate one. However, the association is still noteworthy and may actually be indicative of an important functional variant.

\section{CONFLICT OF INTEREST}

The authors declare no conflict of interest.

\section{ACKNOWLEDGEMENTS}

We thank Angel Garced, Katherine Shimel, and Sun-min Ro, for their assistance in contacting study participants and collecting blood samples. We also thank the study participants and their families for enrolling in this study. This research was supported by the Intramural Research Program of National Eye Institute, NIH and the Australian National Health and Medical Research Council.

1 Friedman DS, O'Colmain BJ, Munoz B et al: Prevalence of age-related macular degeneration in the USA. Arch Ophthalmol 2004; 122: 564-572.

2 Age-Related Eye Disease Study Research G. Risk factors associated with age-related macular degeneration. A case-control study in the age-related eye disease study: agerelated eye disease study report number 3. Ophthalmology 2000; 107: 2224-2232.

3 Age-Related Eye Disease Study Research G. A randomized placebo-controlled, clinica trial of high-dose supplementation with vitamins $C$ and $E$, beta carotene, and zinc for age-related macular degeneration and vision loss: AREDS report no. 8. Arch Ophthalmol 2001; 119: 1417-1436.

4 Clemons TE, Milton RC, Klein R, Seddon JM, Ferris FL III: Risk factors for the incidence of Advanced Age-Related Macular Degeneration in the Age-Related Eye Disease Study (AREDS) AREDS report no. 19. Ophthalmology 2005; 112: 533-539.

5 Evans JR, Fletcher AE, Wormald RP: 28,000 Cases of age related macular degeneration causing visual loss in people aged 75 years and above in the UK may be attributable to smoking. Br J Ophthalmol 2005; 89: 550-553.

6 Hyman L, Neborsky R: Risk factors for age-related macular degeneration: an update. Curr Opin Ophthalmol 2002; 13: 171-175.

7 Ding X, Patel M, Chan CC: Molecular pathology of age-related macular degeneration. Prog Retin Eye Res 2008; 28: 1-18.

8 Chen W, Stambolian D, Edwards AO et al: Genetic variants near TIMP3 and highdensity lipoprotein-associated loci influence susceptibility to age-related macular degeneration. Proc Natl Acad Sci USA 2010; 107: 7401-7406.

$9 \mathrm{Yu}$ Y, Bhangale TR, Fagerness J et al: Common variants near FRK/COL1OA1 and VEGFA are associated with advanced age-related macular degeneration. Hum Mol Genet 2011; 20: 3699-3709.

10 Andreoli MT, Morrison MA, Kim BJ et al: Comprehensive analysis of complement factor $\mathrm{H}$ and LOC387715/ARMS2/HTRA1 variants with respect to phenotype in advanced age-related macular degeneration. Am J Ophthalmol 2009; 148: 869-874.

11 Dewan A, Liu M, Hartman S et al: HTRA1 promoter polymorphism in wet age-related macular degeneration. Science 2006; 314: 989-992.

12 Macgregor AM, Eberhart CG, Fraig M, Lu J, Halushka MK: Tissue inhibitor of matrix metalloproteinase-3 levels in the extracellular matrix of lung, kidney, and eye increase with age. J Histochem Cytochem 2009; 57: 207-213.

13 Kamei M, Hollyfield JG: TIMP-3 in Bruch's membrane: changes during aging and in age-related macular degeneration. Invest Ophthalmol Vis Sci 1999; 40: 2367-2375.

$14 \mathrm{Qi} \mathrm{JH}$, Ebrahem Q, Moore $\mathrm{N}$ et al: A novel function for tissue inhibitor of metalloproteinases-3 (TIMP3): inhibition of angiogenesis by blockage of VEGF binding to VEGF receptor-2. Nat Med 2003; 9: 407-415. 
15 Davis MD, Gangnon RE, Lee LY et al: The Age-Related Eye Disease Study severity scale for age-related macular degeneration: AREDS Report No. 17. Arch Ophthalmol 2005; 123: 1484-1498.

16 Attebo K, Mitchell P, Smith W: Visual acuity and the causes of visual loss in Australia. The Blue Mountains Eye Study. Ophthalmology 1996; 103: 357-364.

17 Mitchell P, Smith W, Attebo K, Wang JJ: Prevalence of age-related maculopathy in Australia. The Blue Mountains Eye Study. Ophthalmology 1995; 102: 1450-1460.

18 Ross RJ, Bojanowski CM, Wang JJ et al: The LOC387715 polymorphism and agerelated macular degeneration: replication in three case-control samples. Invest Ophthalmol Vis Sci 2007; 48: 1128-1132.

19 Maminishkis A, Chen S, Jalickee S et al: Confluent monolayers of cultured human fetal retinal pigment epithelium exhibit morphology and physiology of native tissue. Invest Ophthalmol Vis Sci 2006; 47: 3612-3624.

20 Dudbridge F, Koeleman BP: Efficient computation of significance levels for multiple associations in large studies of correlated data, including genomewide association studies. Am J Hum Genet 2004; 75: 424-435.

21 Fariss RN, Apte SS, Olsen BR, Iwata K, Milam AH: Tissue inhibitor of metalloproteinases-3 is a component of Bruch's membrane of the eye. AmJPathol 1997; 150: 323-328.

22 Della NG, Campochiaro PA, Zack DJ: Localization of TIMP-3 mRNA expression to the retinal pigment epithelium. Invest Ophthalmol Vis Sci 1996; 37: 1921-1924.

23 Weber BH, Vogt G, Wolz W, Ives EJ, Ewing CC: Sorsby's fundus dystrophy is genetically linked to chromosome 22q13-qter. Nat Genet 1994; 7: 158-161.
24 Weber BH, Vogt G, Pruett RC, Stohr H, Felbor U: Mutations in the tissue inhibitor of metalloproteinases-3 (TIMP3) in patients with Sorsby's fundus dystrophy. Nat Genet 1994; 8: 352-356.

25 Felbor U, Stohr H, Amann T, Schonherr U, Weber BH: A novel Ser156Cys mutation in the tissue inhibitor of metalloproteinases-3 (TIMP3) in Sorsby's fundus dystrophy with unusual clinical features. Hum Mol Genet 1995; 4: 2415-2416.

26 Felbor U, Stohr H, Amann T, Schonherr U, Apfelstedt-Sylla E, Weber BH: A second independent Tyr168Cys mutation in the tissue inhibitor of metalloproteinases-3 (TIMP3) in Sorsby's fundus dystrophy. J Med Genet 1996; 33: 233-236.

27 Fariss RN, Apte SS, Luthert PJ, Bird AC, Milam AH: Accumulation of tissue inhibitor of metalloproteinases-3 in human eyes with Sorsby's fundus dystrophy or retinitis pigmentosa. Br J Ophthalmol 1998; 82: 1329-1334.

28 Li Z, Clarke MP, Barker MD, McKie N: TIMP3 mutation in Sorsby's fundus dystrophy: molecular insights. Expert Rev Mol Med 2005; 7: 1-15.

29 Xu P, Liu J, Sakaki-Yumoto M, Derynck R: TACE activation by MAPK-mediated regulation of cell surface dimerization and TIMP3 association. Science Signaling 2012; 5: ra34.

30 Dridi S, Hirano $\mathrm{Y}$, Tarallo $\mathrm{V}$ et al: ERK1/2 activation is a therapeutic target in age-related macular degeneration. Proc Natl Acad Sci USA 2012; 109: $13781-13786$.

31 Brew K, Nagase H: The tissue inhibitors of metalloproteinases (TIMPs): an ancient family with structural and functional diversity. Biochim Biophys Acta 2010; 1803: 55-71. 